\title{
Cause Analysis and Prevention of Hole Collapse by Water Injection and Dust Removal in Qi Panjing Coal Mine
}

\author{
Hua Guo $\mathbb{D}^{1,2}$ Hai-Qiao Wang, ${ }^{1}$ Shi-Qiang Chen, ${ }^{3,4}$ and Zhi-Rong Wu ${ }^{1}$ \\ ${ }^{1}$ School of Resource, Environment and Safety Engineering, Hunan University of Science and Technology, Xiangtan, \\ 411201 Hunan, China \\ ${ }^{2}$ School of Civil and Environmental Engineering, Hunan University of Science and Engineering, Yongzhou, 425199 Hunan, China \\ ${ }^{3}$ Hunan Provincial Engineering Techniques Research Centre of Mine Ventilation \& Dedusting Equipment, Hunan University of \\ Science and Technology, 411201 Hunan, China \\ ${ }^{4}$ Work Safety Key Lab on Prevention and Control of Gas and Roof Disasters for Southern Coal Mines, Hunan University of Science \\ and Technology, Xiangtan, 411201 Hunan, China
}

Correspondence should be addressed to Hua Guo; 160101050001@mail.hnust.edu.cn

Received 25 July 2020; Revised 2 September 2020; Accepted 14 September 2020; Published 20 October 2020

Academic Editor: Bin-Wei Xia

Copyright ( $) 2020$ Hua Guo et al. This is an open access article distributed under the Creative Commons Attribution License, which permits unrestricted use, distribution, and reproduction in any medium, provided the original work is properly cited.

\begin{abstract}
To solve the "special coal seam" with complex coal seam structure, fault phenomenon, and many gangue layers and complex gangue lithology in Qi Panjing coal mine, the problems such as hole collapse and blockage of coal seam water injection dust removal drilling are caused. From the aspects of drilling layout, drilling technology, and gangue material consolidation, a complete set of key technologies for prevention and control of water injection and dust removal in "special coal seam," have been formed. Seven boreholes have been drilled in 1020902 return air roadway of Qi Panjing coal mine, and field comparative test has been carried out. The results show that: after adopting the complete set of key technologies of drilling prevention and control, the drilling depth is $85-100 \mathrm{~m}$, the average depth is $98 \mathrm{~m}$, and the drilling depth of coal seam water injection can reach $170-185 \mathrm{~m}$; the footage per cycle is greatly improved, with the minimum increase of $30.86 \%$, the maximum increase of $46.38 \%$, and the average increase of $36.77 \%$, to save drilling time and bring good economic benefits, and there is no collapse in the borehole hole, to ensure the safety of production. It has a good reference, and practical guiding significance for other coal mining faces, especially for "special coal seam" working face.
\end{abstract}

\section{Introduction}

A lot of dust is produced in the process of mine production, which affects the health of workers, accelerates the wear of machines, pollutes the working environment, and increases the probability of accidents [1]. To reduce the harm caused by dust, scholars at home and abroad have carried out a lot of research work. The dust control of fully mechanized caving face (FMCF) can be divided into dry type and wet type $[2,3]$. The effect of wet dust removal in FMCF is more obvious, and the cost is lower. In all kinds of methods of wet dust removal, coal seam water injection dust removal effect is remarkable, high efficiency, and is a method to prevent and control dust from the root. To achieve the ideal effect, the drilling depth is the key. To ensure that the water injection drilling reaches the design depth, it is necessary to prevent the borehole collapse.

In 2015, Li [4] proposed for the first time the measure of using the inner casing of drill pipe in soft coal seam to solve the hole collapse phenomenon in drilling, compared with the conventional drilling method, and applied to the engineering practice, the effect is remarkable. In 2018, Chen [5] introduced the principle and technology of large-diameter borehole permeability enhancement and applied it to coal seams with complex geological conditions and soft coal quality, laying a good foundation for improving borehole collapse. In 2012, Zhai [6] put forward a new theory that solidified holes are arranged around the construction boreholes, and grouting is carried out in the solidified holes. The slurry flows through the cracks and flows to the construction boreholes 


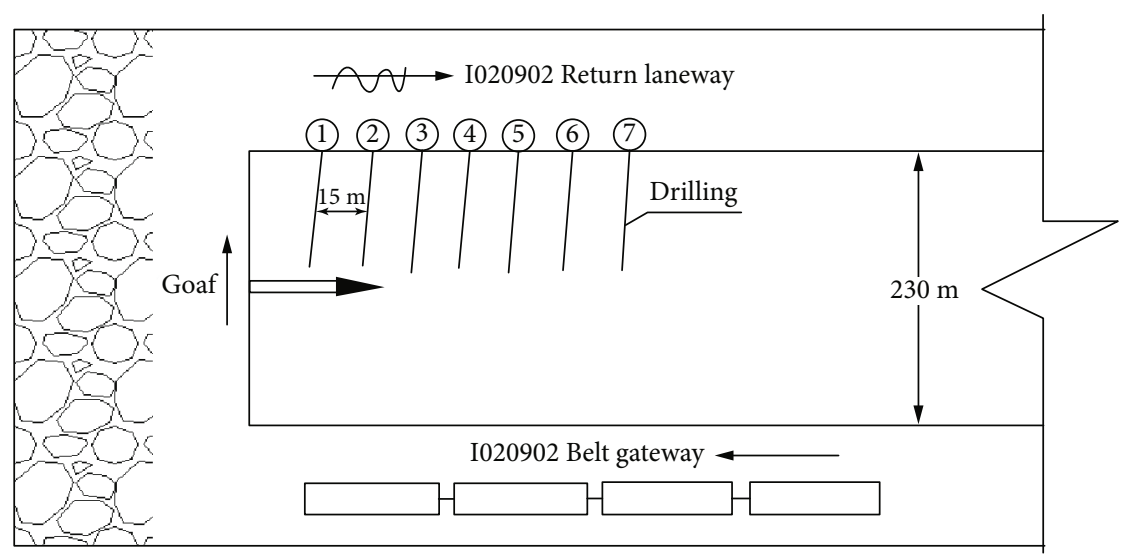

FIGURE 1: Arrangement of drilling for water injection and dedusting in FMCF.

and solidifies, and finally, the construction boreholes are drilled. Also, the stress field and displacement changes around the borehole after the soft coal seam and the solidification with high-strength materials are modelled and analysed. In 1998, Hahn [7] analysed the causes of borehole collapse and put forward a new theory of casing while drilling and setting liner during drilling. The liner and drilling are carried out at the same time to ensure the integrity of drilling, thus establishing a systematic prevention and control system for borehole collapse. Although the above prevention measures have achieved some results, the prevention and control technology of borehole collapse in soft and fractured coal seams is targeted. However, there is little research on the prevention and control of water injection borehole collapse in the "special coal seam" with complex coal seam structure, many faults and gangue layers, and the gangue lithology is mostly clay rock, mudstone, or carbonaceous mudstone. Therefore, given the problem of hole collapse in water injection drilling of "special coal seam" in the Qi Panjing coal mine, the author first analyses the occurrence conditions of coal seam and the causes of hole collapse and then puts forward a complete set of process method of drilling layout, drilling technology combined with gangue material consolidation, forming a systematic water injection dust removal drilling hole collapse prevention technology, which has been applied to engineering practice with remarkable effect.

\section{Cause Analysis of Hole Collapse in Water Injection Dust Removal Drilling}

2.1. FMCF of Water Injection Dedusting. The natural thickness of the coal seam of I020902 FMCF for coal seam water injection in Qi Panjing coal mine is $2.18-8.68 \mathrm{~m}$, with an average of $4.19 \mathrm{~m}$. The reserve utilisation thickness is $1.70-$ $5.31 \mathrm{~m}$, with an average of $2.91 \mathrm{~m}$. The coal seam is thin in the East and thick in the West. There are 1-8 layers of gangue in the whole coal seam, most of which are 4-5 layers. The main lithology of gangue is a clay rock, mudstone, or carbonaceous mudstone. The coal seam of I020902 FMCF is difficult to clean coal with medium ash content, low sulfur content, low medium phosphorus coal, high volatile matter, medium-high calorific value, and medium clean coal recovery rate. The coal quality grade is $1 / 3 \mathrm{JM}$, which belongs to soft coal with more fractures. I020902 FMCF elevation $+800-913 \mathrm{~m}$, FMCF length of $230 \mathrm{~m}$, coal seam affusion drilling design length of $170 \mathrm{~m}$, and ZYWL-3200 crawler type drilling rig are selected. The drilling rig is matched with the drill pipe and head. The pulverised coal particles in the drilling hole are discharged by water flushing. After drilling for some time, the drilling team drilled seven boreholes. Generally, the hole collapse occurs at the length of $70-80 \mathrm{~m}$, as shown in Figure 1, which is far from the designed, so it is impossible to inject water into the long hole coal seam.

\subsection{Stress Analysis of Water Injection Dedusting Borehole.} The stress state around the water injection dust removal borehole in "special coal seam" is the decisive factor for whether the borehole collapses. With the drilling of the borehole, the stress balance is destroyed due to the application of redistribution around the borehole. According to the stress direction, there are radial stress, tangential stress, and drilling direction stress $[8,9]$. The stress size is affected by coal seam buried depth, geological structure, mechanical properties of coal, and drilling characteristics. According to the different distances between the stress-strain of coal and the borehole, it can be divided into an elastic zone, plastic zone, and fracture zone. When it is in the elastic zone, the coal body around the borehole is far away from the borehole and fails to reach the plastic deformation condition; when it is in the plastic zone, the coal body around the borehole is close to the borehole to reach the plastic deformation condition, but it does not cause fracture deformation and is in the plastic state; When it is in the fracture zone, the coal body around the borehole reaches the plastic deformation condition and causes fracture deformation.

It is assumed that there is ideal elastic-plastic material around the borehole of the coal body [10], and it is incompressible. Because the length of the borehole is far larger than the diameter of the borehole, it can be regarded as a plane stress problem for analysis. The effective stress in 


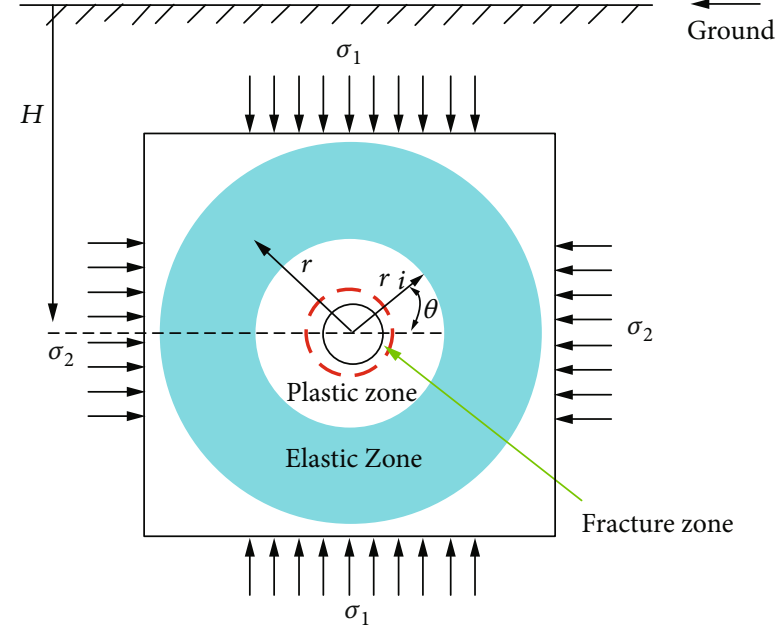

FIGURE 2: Stress state of water injection dust removal borehole in coal body.

"special coal seam" is the difference between coal stress and coal pore pressure, and its expression is

$$
\delta_{e}=\delta-\alpha p,
$$

where $\delta$ is the stress of coal mass $(\mathrm{MPa}), \alpha$ is the effective stress coefficient of special coal, $\alpha=C_{0} / C$, which is generally 0.4-0.7 as soft coal, and $p$ is pore pressure (MPa).

(1) Stress in the elastic zone of coal seam water injection dust removal borehole

The stress state of water injection dust removal borehole in the coal body is shown in Figure 2. Without considering the seepage of the hole wall, under the combined action of radial stress, tangential stress, drilling direction stress, and pore pressure [11-13], the effective stress expression of the elastic zone around the hole wall is as follows:

$$
\begin{aligned}
\delta_{r}= & \frac{\delta_{1}+\delta_{2}}{2}\left(1-\frac{r_{i}^{2}}{r^{2}}\right)+\frac{\delta_{1}-\delta_{2}}{2}\left(1-4 \frac{r_{i}^{2}}{r^{2}}+3 \frac{r_{i}^{4}}{r^{4}}\right) \cos 2 \theta \\
& +\frac{r_{i}^{2}}{r^{2}} p_{1}-\alpha p,
\end{aligned}
$$

$$
\begin{aligned}
\delta_{\theta}= & \frac{\delta_{1}+\delta_{2}}{2}\left(1+\frac{r_{i}^{2}}{r^{2}}\right)-\frac{\delta_{1}-\delta_{2}}{2}\left(1+3 \frac{r_{i}^{4}}{r^{4}}\right) \cos 2 \theta \\
& -\frac{r_{i}^{2}}{r^{2}} p_{1}-\alpha p,
\end{aligned}
$$

$\delta_{z}=\delta_{a}-2 \mu\left(\delta_{1}-\delta_{2}\right) \frac{r_{i}^{2}}{r^{2}} \cos 2 \theta-\alpha p$

$$
\tau_{r \theta}=\frac{\delta_{1}-\delta_{2}}{2}\left(1-3 \frac{r_{i}^{2}}{r^{2}}+2 \frac{r_{i}^{4}}{r^{4}}\right) \sin 2 \theta,
$$

where $C_{0}$ is the skeleton compressibility of coal, $C$ is volume compressibility of coal, $\delta_{r}$ is radial effective normal stress
(MPa), $\delta_{\theta}$ is circumferential effective normal stress (MPa), $\delta_{z}$ is drilling direction effective normal stress $(\mathrm{MPa}), \tau_{r \theta}$ is shear stress $(\mathrm{MPa}), \delta_{1}$ is vertical maximum principal in-situ stress $(\mathrm{MPa}), \delta_{2}$ is horizontal maximum principal in-situ stress $(\mathrm{MPa}), \delta_{\alpha}$ is upper pressure of original rock (MPa), $\theta$ is the angle between the vector diameter of the point on the hole wall and the maximum horizontal principal geostress $\left({ }^{\circ}\right), r_{i}$ is drilling radius $(\mathrm{m})$, and $r$ is the distance from drilling centre line $(\mathrm{m})$.

(2) Stress in the plastic zone of coal seam water injection dust removal borehole

According to the division of the stress area around the borehole, assuming that the coal mass is incompressible and $r=r_{i}$, the stress distribution in the plastic zone is as follows:

$$
\begin{aligned}
\delta_{r} & =p_{1}-\alpha p, \\
\delta_{\theta} & =\delta_{1}(1-2 \cos 2 \theta)+\delta_{2}(1+2 \cos 2 \theta)-p_{1}-\alpha p, \\
\delta_{z} & =\delta_{a}-2 \mu\left(\delta_{1}-\delta_{2}\right) \cos 2 \theta-\alpha p, \\
\tau_{r \theta} & =0,
\end{aligned}
$$

where $p_{1}$ is the minimum wall support pressure (gas pressure in the hole for soft special coal seam and slag removal by wind) $(\mathrm{MPa})$.

(3) Failure pressure of coal seam water injection dust removal borehole

As long as the strength of the borehole itself is lower than the stress of the coal around the borehole, the shear failure will occur, and the hole collapse will occur [14]. According to the mechanical analysis of borehole wall stability, combined with the Mohr-Coulomb criterion, the effective stress can be expressed as follows:

$$
\delta_{\max }=\delta_{\min } \frac{1+\sin \phi}{1-\sin \phi}+2 C \frac{\cos \phi}{1-\sin \phi},
$$

where $\delta_{\max }$ is the maximum principal stress of the hole wall $(\mathrm{MPa}), \delta_{\min }$ is the minimum principal stress of the hole wall $(\mathrm{MPa}), \phi$ is the internal friction angle of the coal body $\left({ }^{\circ}\right)$, and $\mathrm{C}$ is the cohesion of the coal body (MPa).

From the above formula, it can be seen that the internal friction angle and cohesion of the coal body are related to the mechanical properties of coal, which are fixed constant values; the shear failure of the coal body is mainly controlled by the maximum and minimum principal stress of hole wall, and the greater the difference between $\delta_{\max }$ and $\delta_{\min }$, the easier the hole wall collapses. According to the analysis of the principal stress of coal drilling, the horizontal stress distribution around the borehole is not uniform, and the stress $\left(\delta_{\theta}\right)$ around the borehole changes with the change of $\theta$ angle. The maximum principal stress of the coal body can be approximately $\delta_{\theta}$, and the minimum principal stress can be approximately $\delta_{r}$. When $\theta=90^{\circ}$ or 270 , the effective stress 
difference $\delta_{\theta}-\delta_{r}$ is the largest, which is most likely to cause borehole collapse. At this time, the effective stress formula of borehole collapse is as follows:

$$
\begin{aligned}
& \delta_{r \mid r=r_{i}}=p_{1}-\alpha p_{1}, \\
& \delta_{\theta \mid r=r_{i}}=\eta\left(3 \delta_{1}-\delta_{2}-p_{1}\right)-\alpha p_{1}, \\
& \tau_{\left.r \theta\right|_{r=r_{i}}}=0,
\end{aligned}
$$

where $\eta$ is the nonlinear correction coefficient.

Substituting (11) and (12) into equation (10) and making $k=\cot \left(45^{\circ}-\phi / 2\right)$, the collapse pressure of the hole wall is obtained as follows:

$$
p_{1}=\frac{\eta\left(3 \delta_{1}-\delta_{2}\right)-2 C k+\alpha p_{1}\left(k_{2}-1\right)}{k^{2}+\eta} .
$$

2.3. Concrete Analysis of Hole Collapse. According to the occurrence and geological conditions of the coal seam in Qi Panjing coal mine, the water injection feasibility of the coal seam sampling in Qi Panjing coal mine is analysed. The original moisture value of the coal seam is $2.38 \%$. The average total porosity of the coal seam is $6.42 \%$ calculated from the dry block density and true density of the test coal body. The natural water absorption rate of the coal is $1.305 \%$, the firmness coefficient of the coal seam is 0.6848 , which is compared with the standard value of the water injection injectability of the coal seam, it can be concluded that the coal seam is water injectable [15-17]. Combined with the complexity of water injection technology, roadway layout, and water injection effect, long borehole water injection is finally adopted.

From the beginning of drilling to the depth of $70 \mathrm{~m}$, it is normal. After 70-80 m, the backwater in the hole decreases, the hole collapse occurs, and the load of the drilling rig increases. After slowing down and punching, the drilling continues, and the drilling is difficult. Under the circumstances of taking measures such as stopping drilling, punching, and reverse reciprocating hole cleaning, there is still no practical effect. After analysis, the specific reasons are as follows:

(1) This coal seam is soft, with faults, many fractures, and many gangue layers. To avoid the position of faults and fractures, the middle and upper part of the coal seam in the air return roadway of the working face shall be drilled for water injection from a certain angle to the advancing direction of the FMCF, or the middle and upper part of the FMCF shall be transported by the belt of the FMCF to the advancing direction of the FMCF at a certain angle. Considering that the pipeline and equipment are arranged in the belt conveyor roadway of the FMCF, the drilling water injection is carried out in the middle and upper part of the coal seam of the return air roadway of the FMCF perpendicular to the advancing direction of the FMCF. Due to the influence of driving on both sides of the roadway, the stress concentration area is formed, and the coal body cracks are relatively developed. During the long hole drilling, affected by the pressure, the coal seam will give out a "click" sound during the drilling process. Then, the hole collapse phenomenon will occur, blocking the normal borehole backwater

(2) There is an SF12 normal fault in the scope of I020902 FMCF, which is located in the centre of $1200 \mathrm{~m}$ of FMCF. It is an East-West strike; the dip angle is $65^{\circ}$, and the drop is $5 \mathrm{~m}$. The strike extension length is about $400 \mathrm{~m}$. The fault position is accurately controlled, but hole collapse will occur when the fault is encountered during drilling

(3) During drilling, the gangue in the coal seam will be encountered when drilling to $70 \mathrm{~m}$. The gangue is mainly claystone, mudstone, or carbonaceous mudstone, with argillaceous cementation, low hardness, and loose sand grain when meeting water. After analysis and research, the hole wall cannot form an effective whole, but loose, and hole collapse occurs

\section{Technical Measures for Hole Collapse Prevention}

\subsection{Drilling Site Selection}

(1) The drilling location is the place with poor development of coal seam fissures. The drilling angle is arranged according to the coal seam strike and dips angle. The water injection hole is arranged at $1.8 \mathrm{~m}$ away from the coal seam floor to avoid the sinking of the drill pipe and penetrating the floor when the long hole drill pipe is long

(2) Accurately judge the fault within the scope of I020902 working face, select the position that can avoid the fault, and ensure that the drilling does not pass through the fault affected area

(3) When passing through the stress concentration area on one side of the roadway, the drilling speed should be appropriately slowed down, and the "feed pressure" of drilling should be reduced to maintain the normal drilling backwater state. After passing through the stress concentration area, the normal drilling speed should be restored. When drilling down from the middle and upper part of the coal seam perpendicular to the advancing direction of the FMCF, the drill pipe will speed up the drilling speed due to its weight, so the drilling speed should be controlled properly

3.2. Drilling Arrangement. In deep hole drilling, drilling design plays a fundamental role. In the coal seam, the specific drilling position and drilling angle play an important role [18-22]. According to the coal seam thickness of I020902 FMCF, combined with roadway height, borehole inclination angle, and water movement along joint fissure after water injection, it cannot be close to the lower part of the coal seam, 


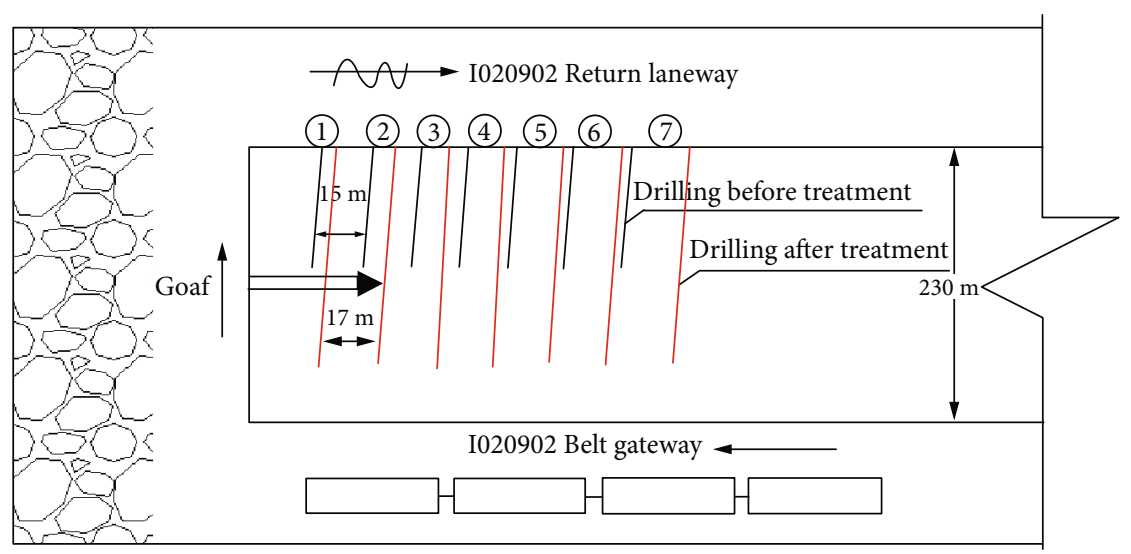

FIgURE 3: Comparison of the layout before and after dedusting drilling treatment.

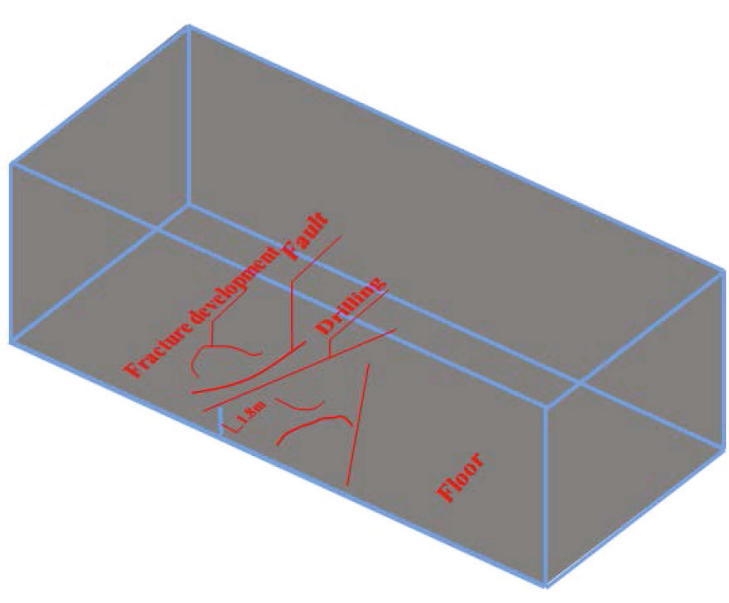

FIgURE 4: Details of dedusting drilling.

to avoid side slope phenomenon of lower FMCF after water injection. The above factors are considered comprehensively. Finally, it is determined that the drilling direction is nearly perpendicular to the main body of the coal seam, and the height from the coal seam floor is $1.8 \mathrm{~m}$, the drilling angle is divided into the upward hole and downward hole [23]. After analysing the specific conditions of the FMCF, the drilling angle of the upward hole should be less than $2^{\circ}$ to $3^{\circ}$ of the coal seam inclination angle. The down hole drilling angle should be $1^{\circ}$ to $2^{\circ}$ greater than the coal seam dip angle, to ensure the drilling track trace basically goes along the coal seam [24-26], which can increase the drilling depth.

3.3. Drilling Tool Selection. To reduce the occurrence of hole collapse during drilling, the long conical bit should be considered. Compared with the ordinary drill bit, the long conical bit has the following characteristics: the head is sharp and can eat into the coal seam well. First, form a small area of drilling, and then through the middle and bottom of the long cone drill bit to expand the hole diameter required for coal seam water injection. Because this kind of drill bit is easy to drill holes, with good hole shape and fine coal powder, it can be discharged well with the drilling machine, so as not to block the drilling hole. On the premise of meeting the requirements of water injection drilling hole diameter, properly reducing the drilling hole diameter can reduce the hole collapse phenomenon caused by the pressure on the deep coal seam due to the large drilling area.

When drilling, we usually use high-pressure water to flush out the pulverised coal in the drilling hole. Sometimes, some pulverised coal and particles in the hole cannot be discharged. In this case, if we continue to use high-pressure water to wash the coal powder, the water pressure in the borehole will increase suddenly, resulting in the stress concentration of the newly drilled wall, which will damage the newly drilled hole, in the case of larger area collapse. The drill pipe with pulse water flushing and hollow screw is designed, that is, there are large alternate threads on the drill pipe. During normal drilling, the long conical bit reduces the occurrence of hole collapse. Even if the hole collapse occurs, the amount of hole collapse is greatly reduced. The back end spiral drill pipe can effectively discharge the coal powder and particles produced.

3.4. Drilling Technology and Method. During drilling, the drilling rig must be stable. It is to connect the drilling rig and the ground as a stable whole. Solid wood can be padded on the bottom of the drilling rig, and the position of the drilling rig can be firmly controlled with columns to avoid vibration during drilling. Once the vibration occurs, the drilling eccentricity will be formed, and the hole wall will be uneven, the drilling resistance will be increased, and the drilling capacity of the drilling rig will be virtually weakened, which will affect the drilling effect. The probability of drilling hole plugging is largely promoted.

During drilling, the relationship between pressure and speed should be grasped. The limit drilling pressure is fixed in the drilling process of each drilling rig, but the pressure given by layers with different hardness is different. It is necessary to analyse the specific layers and pay attention to the changes of pressure during layer change, withdrawal, and pull out the drilling. When changing the layer, it is easy to block the hole and damage the drilling tool, resulting in the pressure change of the whole drilling device. It needs to be observed at any time. Once the pressure rises, the drilling should be stopped in time for mucking. The drilling speed 


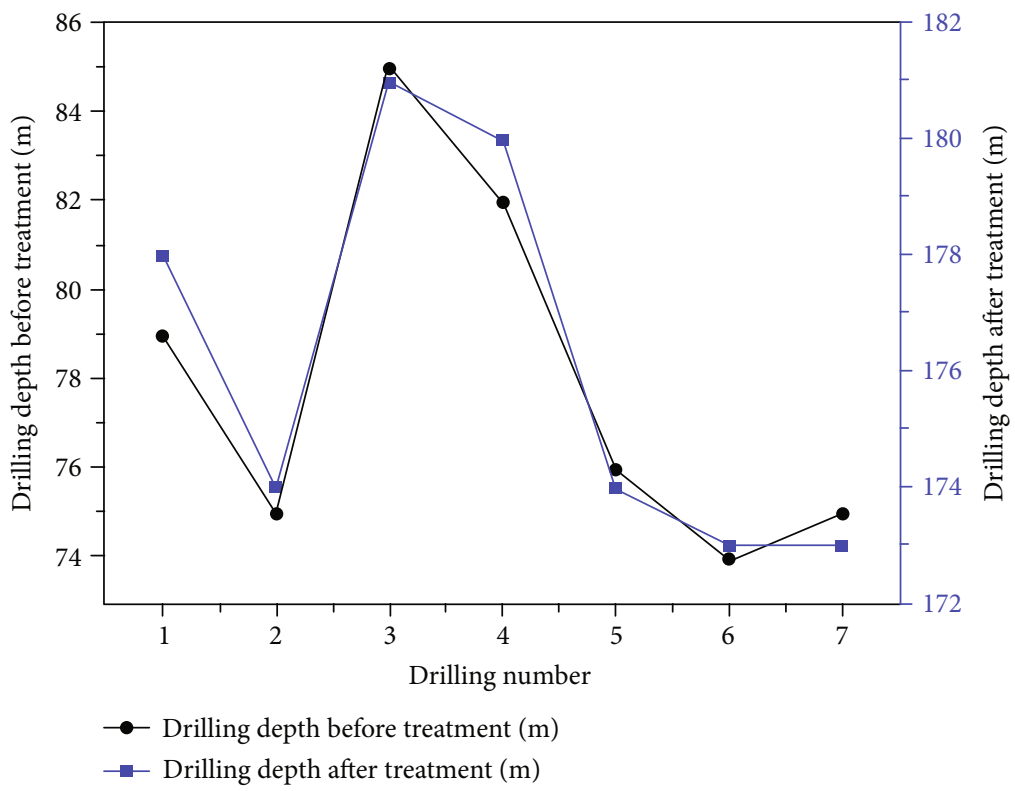

FIgUre 5: Comparison of dedusting drilling depth.

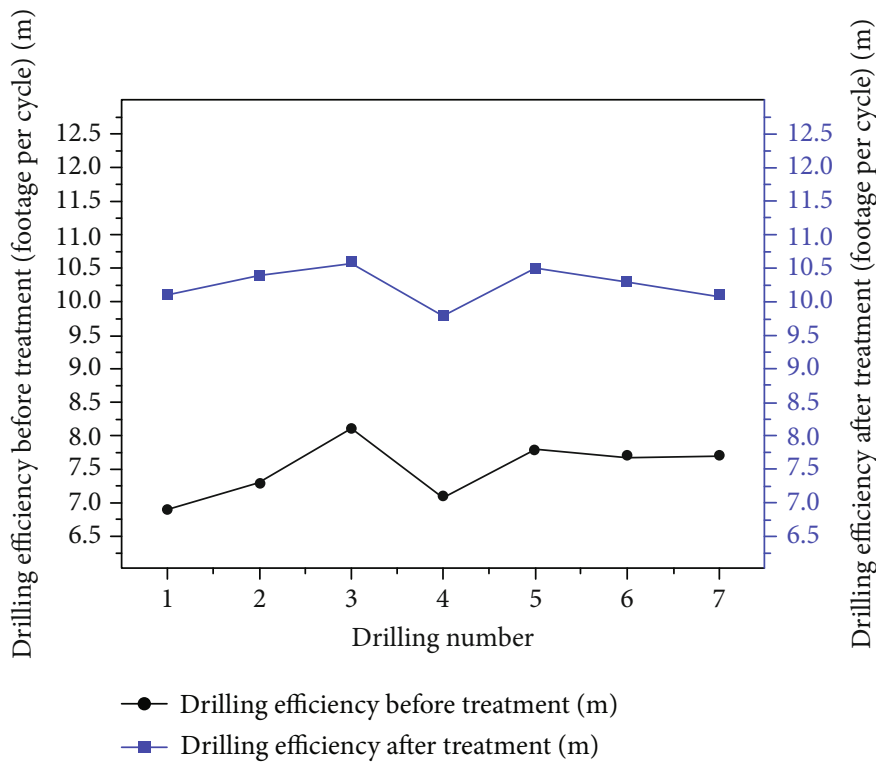

Figure 6: Comparison of dedusting drilling efficiency.

should not be too fast when withdrawal and pulling out the drilling. To maintain the appropriate speed and grasp the strength, experienced drilling operators are required to make a reasonable judgment on the slag discharge conditions and drilling speed according to the summary.

Ensure that drilling operators have high drilling skills. People have an important impact on the quality of drilling rig drilling, especially the technological level of drilling personnel is the decisive factor of whether the drilling can achieve the expected quality, which requires the professional level training of drilling operators and the implementation of the system so that they all have professional technical knowl- edge. The operators can not only drill but also repair the drilling rig, know how to operate the rig efficiently and normally, and can deal with emergencies.

3.5. Material Consolidation Method. In the process of drilling, encountered in the coal seam gangue [27, 28], the measures taken are water glass chemical grouting material grouting consolidation, the original collapse hole filled with water glass chemical grouting material, so that the sediment in the hole and sodium silicate chemical grouting material mixed, can be better cemented into a whole. It can effectively fill the collapse hole space and solidify the collapse hole wall. 
According to the actual situation and repeated discussion and analysis, although this method is not used in coal seam water injection drilling hole collapse treatment, it has been used in other temporary or semipermanent engineering projects, and the effect is good. Considering its good profitability, low cost, and high safety coefficient, this method is adopted. In this method, water glass, ethylene glycol adipate, Ma additive, and water are mixed in the proportion of $50: 2: 6: 42$ to form grouting raw materials. The mud pump is used to pour the bottom of the drill pipe to the collapsed hole. Starting from the lowest end of the hole collapse, the drill pipe is moved back and forth during grouting, so that the loose sediment at the collapsed hole floats with the sodium silicate chemical grouting material under the impact of water glass chemical grouting material, forming the whole structure. After the water in the hole is discharged and the water glass chemical grouting material flows out of the hole, stop the grouting and trip out, clean the drilling tool. After 48 hours, the hole can be swept after the grouting material, and the sediment in the hole are solidified.

\section{Results and Discussion}

This paper analyses the reasons for the hole collapse of water injection dust removal borehole in I020902 FMCF, and after taking relevant technical measures, the ZYWL-3200 crawler drilling rig is used for drilling. The comparison layout position before and after drilling treatment is shown in Figure 3, and the detailed hole drawing after hole collapse prevention technology is shown in Figure 4. The comparison data before and after the drilling depth is shown in Figure 5. Through the analysis of Figure 5, the drilling depth of the seven boreholes after treatment is increased by $85-100 \mathrm{~m}$, the average depth is increased by $98 \mathrm{~m}$, and the drilling depth of water injection in coal seam can reach $170-185 \mathrm{~m}$, which indicates that the treatment measures are appropriate. The treatment effect has reached the expectation. Also, when the coal seam water injection drilling operation was started, the drilling team made a footage of 7 meters per cycle, and then the borehole collapse occurred. Corresponding measures were taken to control the drilling efficiency, and the drilling efficiency was also greatly improved. The detailed data and trends are shown in Figure 6. Through the analysis of Figure 6, it can be seen that after treatment, the footage per cycle has been greatly improved, with a minimum increase of $30.86 \%$, a maximum increase of $46.38 \%$, and an average increase of $36.77 \%$, thus saving valuable time and bringing greater economic benefits. Also, there is no hole collapse phenomenon in water injection dust removal drilling, which ensures the safety of production.

\section{Conclusion}

(1) Based on the analysis of the causes of hole collapse in water injection dust removal drilling, the paper puts forward the new technology of systematic prevention and control of hole collapse in water injection dust removal drilling hole: from the selection of drilling site, drilling layout, drilling tool selection, determina- tion of drilling technology, and other aspects, the treatment measures are put forward. Especially in case of hole collapse caused by gangue in the coal seam, water glass grouting material is adopted for grouting consolidation. After reaching a certain strength, the hole shall be drilled again. This has great reference significance and popularisation value for the prevention and control of hole collapse of water injection dust removal drilling hole in another coal mine FMCF

(2) For the coal seam with complex coal seam structure, many gangue layers, and complicated gangue lithology, seven water injection dust removal boreholes were drilled. The borehole collapse occurred, and the prevention measures were taken. The drilling depth was $85-100 \mathrm{~m}$ higher than the original, with an average increase of $98 \mathrm{~m}$, and the water injection drilling depth of the coal seam could reach 170-185 m

(3) The drilling team's footage per cycle was 7 meters, and then the borehole collapse occurred. After treatment, the footage per cycle increased greatly, at least by $30.86 \%$, the maximum increase of $46.38 \%$, and the average increase of $36.77 \%$. The drilling time was saved, and the economic benefit was good. Moreover, there was no hole collapse in the drilling, which ensured the production safety

\section{Data Availability}

All data included in this study are available upon request by contact with the corresponding author.

\section{Conflicts of Interest}

The authors declare no conflict of interest.

\section{Acknowledgments}

This work was conducted with support from the 2017 Hunan Provincial Graduate Research Innovation Project of China (No. CX2017B649), the Excellent youth project of Hunan Provincial Department of Education (No. 19B223), National Natural Science Foundation of China (No. 51774134), and General scientific research projects of Hunan University of Science and Engineering (No. 19XKY056).

\section{References}

[1] D. W. Li, J. Ma, and H. Q. Liu, Prevention and Control of Dust and Occupational Diseases in Coal Mines, China University of Mining and Technology Press, Xuzhou, 2007.

[2] Y. Peng, Z. A. Jiang, E. Q. Fu, S. Han, and G. Lan, "Study onseam water injection and dust control optimisation and effect of fully-mechanized coal mining face," Coal Science and Technology, vol. 46, no. 1, pp. 224-230, 2018.

[3] T. Liu, S. Y. Lan, C. M. Wang, and E. Z. Lv, "Comparative study on application of high-pressure spray and foam dust removal technology in fully mechanized heading face," Mining 
Safety \& Environmental Protection, vol. 43, no. 1, pp. 50-53, 2016.

[4] D. Q. Li, G. T. Deng, H. G. Li, and M. Z. Wang, "Technology of preventing drilling hole collapse in soft coal seam by inserting casing in drill pipe," Journal of Safety Science and Technology, vol. 11, no. 2, pp. 95-98, 2015.

[5] J. F. Chen, J. Z. Wei, G. J. Zhang, and L. Wang, "Study on permeability improvement technology of the large-diameter drilling combined with hole protection," Coal Science and Technology, vol. 46, no. 10, pp. 73-77, 2018.

[6] C. Zhai, Q. G. Li, and C. Su, "Analysis on borehole instability and control method of pore-forming of hydraulic fracturing in soft coal seam," Journal of China Coal Society, vol. 37, no. 9, pp. 1431-1433, 2012.

[7] D. Hahn, F. Makohl, and L. Watkins, "Casing-while-drilling system reduces hole collapse risks," Offshore, vol. 58, no. 2, p. 54, 1998.

[8] L. G. Zhang, X. P. Sun, S. B. Li, and Y. X. Li, Fundamentals and applications of rock mechanics, Harbin Institute of Technology Press, Harbin, 2016.

[9] Z. Wang, Y. P. Liang, and H. W. Jin, "Analysis of mechanics conditions for instability of outburst-preventing borehole," Journal of Mining \& Safety Engineering, vol. 25, no. 4, pp. 444-448, 2008.

[10] E. Papamichos, "Analysis of borehole failure modes and pore pressure effects," Computers and Geotechnics, vol. 37, no. 1-2, pp. 141-152, 2010.

[11] T. Meier, E. Rybacki, A. Reinicke, and G. Dresen, "Influence of borehole diameter on the formation of borehole breakouts in black shale," International Journal of Rock Mechanics and Mining Sciences, vol. 62, pp. 74-85, 2013.

[12] A. Patutin and S. Serdyukov, "Transverse hydraulic fracture initiation by indentation in an uncased borehole," Procedia Engineering, vol. 191, pp. 287-290, 2017.

[13] J. Ptáček, P. Konicek, L. Staš, P. Waclawik, and R. Kukutsch, "Rotation of principal axes and changes of stress due to mine-induced stresses," Canadian Geotechnical Journal, vol. 52, no. 10, pp. 1440-1447, 2015.

[14] L. G. Zhang, "Borehole stability analysis accounting for anisotropies in drilling to weak bedding planes," International Journal of Rock Mechanics and Mining Sciences, vol. 60, pp. 160170, 2013.

[15] H. Guo, Study on Technological Method of Water Injection in Coal Seam, Kunming University of Science and Technology, Kunming, 2014.

[16] S. Hu, B. Si, H. Shu, and X. Song, "Fisher information of mine collapse hole detection based on sensor nodes connectivity," International Journal of Advancements in Computing Technology, vol. 5, no. 9, pp. 1245-1254, 2013.

[17] L. Huang, Y. Y. Lu, B. W. Xia, Y. J. Jia, and F. Huang, "Elastoplastic analysis of surrounding rock of drilling with strain softening model in deep soft rock," Rock and Soil Mechanics, vol. 34, Supplement 1, pp. 179-186, 2013.

[18] X. R. Yao, G. L. Chen, and B. M. Shi, "Analysis on gas extraction drilling instability and control method of pore-forming in deep surrounding-rock with a weak structure," Journal of China Coal Society, vol. 35, no. 12, pp. 2073-2081, 2010.

[19] S. X. Chen, F. Yang, and J. J. Zhang, "Key technology of offshore drilling for gold mine in the northern sea area of Sanshandao," Gold Science and Technology, vol. 24, no. 1, pp. 17-22, 2016.
[20] Q. H. Ji, "Research and application of auger-air drilling and sieve tube borehole protection in soft outburst-prone coal seams," Procedia Engineering, vol. 73, pp. 283-288, 2014.

[21] Z. Zhang, Y. X. Sun, Y. K. Fu, and T. Wang, "Study and application of pre-reinforcement of soft and breaking coal mass in gas drainage drilling field," Coal Engineering, vol. 51, no. 8, pp. 44-47, 2019.

[22] T. Li, Y. Gao, D. C. Al, J. W. Yang, H. Feng, and G. J. Zhao, "Floor precise grouting for prevention and control of waterbased on confined water single-hole drainage experiment," Journal of China Coal Society, vol. 44, no. 8, pp. 2494-2501, 2019.

[23] Z. Q. Wu, "Treatment of drilling collapse by water injection in deep hole coal seam," Shandong Coal Science and Technology, no. 1, pp. 15-16, 2007.

[24] Z. X. Dai, H. Q. Chen, and S. C. Zheng, "Innovation and application of deep hole drilling technology in outburst soft coal seam," Safety in Coal Mines, vol. 38, no. 8, pp. 20-21, 2007.

[25] W. He, X. W. Zhou, and R. Xu, "Experimental research on the new type of sodium silicate chemical grouting material," Journal of China Coal Society, vol. 36, no. 11, pp. 1812-1815, 2007.

[26] H. Z. Zhang and X. C. Wang, "The treatment process of ZK0702 drilling accident in Baoxinggou gold mine, Heilongjiang province," Gold Science and Technology, vol. 19, no. 5, pp. 53-55, 2011.

[27] T. Li, Y. Gao, P. Zhang et al., "Study on modification of grouting material with clay-based plate in deep mining under pressure," Metal Mine, vol. 522, no. 12, pp. 173-177, 2019.

[28] H. Guo, H. Q. Wang, and J. Zhou, "Study on coal seam water infusion technology in fully mechanized coal face of Qipanjing mine," Mining Safety \& Environmental Protection, vol. 44, no. 5, pp. 14-17, 2017. 